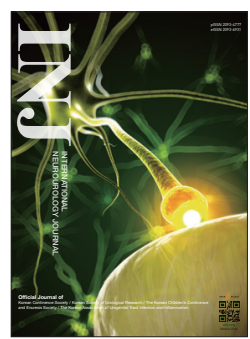

\title{
At the End of the Year 2019
}

\author{
Khae Hawn Kim (iD https://orcid.org/0000-0002-7045-8004 \\ Department of Urology, Gachon University Gil Medical Center, Gachon University of School of Medicine, Incheon, Korea \\ E-mail:kimcho99@gilhospital.com
}

The year of 2019 is ending, leaving behind many stories and a history of events. The new year of expectation and hope is coming here. I would like to greet those involved in the publication of International Neurourlogy Journal (INJ) and those who contribute papers to INJ.

Merry Christmas and Happy New Year.

In this year, the INJ has also published many papers. We published four regular papers and 2 supplements in 2019. The first supplement subject was "Mitochondrial Dysfunction" and the second supplement subject was "Neuroinflammation." The reason for publishing supplements was to broaden access to neurourology. INJ considers the first purpose of overcoming urological diseases, but furthermore, it is intended to examine urological diseases by connecting the nervous system.

Integrated coordination of the lower urinary tract components is mediated by the complex nervous control systems of the brain, spinal cord, and ganglia. Therefore, the nervous system plays a decisive role in the storage and output of urine. The purpose of the INJ is to present not only the main aspects of the structure of the nervous system associated with the urinary tract, but also the mechanisms of action that regulate the storage and output of urine and the control system of nerve distribution. The connection with the nervous system shows the cause of urinary diseases more clearly, and I think that it has opened a new way to treat urological diseases.

It was more concerned with the management of impact factor, and we tried INJ to be introduced a lot in other journals. Connecting different fields into one theme is a unique merit of INJ. These attempts will help you find new ways when the problem is not solved. The originality and novelty of INJ is worth mentioning in other journals.

I look forward to further development of the INJ in the area of neurourology. I express my sincere gratitude to the authors who have contributed valuable papers.

- Conflict of Interest: No potential conflict of interest relevant to this article was reported. 